\title{
Environmental enrichment requires adult neurogenesis to facilitate the recovery from psychosocial stress
}

\author{
RJ Schloesser ${ }^{1,3}, \mathrm{M}$ Lehmann ${ }^{2,3}, \mathrm{~K}$ Martinowich${ }^{1}, \mathrm{HK}$ Manji ${ }^{1}$ and M Herkenham² \\ ${ }^{1}$ Laboratory of Molecular Pathophysiology, National Institute of Mental Health (NIMH), National Institutes of Health (NIH), \\ Bethesda, MD, USA and ${ }^{2}$ Section on Functional Neuroanatomy, National Institute of Mental Health (NIMH), \\ National Institutes of Health (NIH), Bethesda, MD, USA
}

\begin{abstract}
The subgranular zone of the adult hippocampal dentate gyrus contains a pool of neural stem cells that continuously divide and differentiate into functional granule cells. It has been shown that production of new hippocampal neurons is necessary for amelioration of stress-induced behavioral changes by antidepressants in animal models of depression. The survival of newly born hippocampal neurons is decreased by chronic psychosocial stress and increased by exposure to enriched environments. These observations suggest the existence of a link between hippocampal neurogenesis, stress-induced behavioral changes, and the beneficial effects of enriched environment. To show causality, we subjected transgenic mice with conditionally suppressed neurogenesis to psychosocial stress followed by environmental enrichment. First, we showed that repeated social defeat coupled with chronic exposure to an aggressor produces robust and quantifiable indices of submissive and depressive-like behaviors; second, subsequent exposure to an enriched environment led to extinction of the submissive phenotype, while animals exposed to an impoverished environment retained the submissive phenotype; and third, enrichment was not effective in reversing the submissive and depressive-like behaviors in transgenic mice lacking neurogenesis. Our data show two main findings. First, living in an enriched environment is highly effective in extinguishing submissive behavioral traits developed during chronic social stress, and second, these effects are critically dependent on adult neurogenesis, indicating that beneficial behavioral adaptations are dependent on intact adult neurogenesis.
\end{abstract}

Molecular Psychiatry (2010) 15, 1152-1163; doi:10.1038/mp.2010.34; published online 23 March 2010

Keywords: hippocampal neurogenesis; social stress; environmental enrichment; dentate gyrus; anxiety; depression

\section{Introduction}

Environmental enrichment (EE)-the addition of tubes, ladders and running wheels in an enlarged home environment-enhances cognitive, motor and sensory function. EE has beneficial physiological and behavioral effects in animal models of neurodegenerative disease and psychiatric disorders. ${ }^{1}$ Importantly, EE can ameliorate emotional disturbances induced by psychological stress. ${ }^{2}$

Stressful life events have been established as precipitating and/or exacerbating factors in the development of disturbed mood. ${ }^{3,4}$ Several lines of evidence support a role for adult hippocampal neurogenesis in the regulation of stress-related affective disorders. Stress exposure decreases ${ }^{5,6}$ and antidepressant

Correspondence: Dr M Lehmann, Section on Functional Neuroanatomy, National Institute of Mental Health, Building 35, Room 1C911, 35 Convent Drive, Bethesda, MD 20892, USA.

E-mail: michael.lehmann@nih.gov

${ }^{3}$ These authors contributed equally to this work.

Received 30 July 2009; revised 28 January 2010; accepted 16 February 2010; published online 23 March 2010 treatment increases hippocampal neurogenesis., Importantly, certain antidepressant-induced behavioral changes following stress exposure are dependent on intact hippocampal neurogenesis. ${ }^{9,10}$

In the subgranular zone (SGZ) of the hippocampal dentate gyrus (DG), newly generated cells ${ }^{11-13}$ migrate into the DG granule cell layer where they differentiate into mature neurons and integrate into the existing hippocampal circuitry. ${ }^{14,15}$ Accumulating evidence from behavioral analyses suggests a functional role for hippocampal adult neurogenesis in some forms of learning, memory and mood regulation. ${ }^{9,16-20}$

EE is a strong inducer of hippocampal neurogenesis. In addition, EE confers stress resilience, tempering the animal's emotional reactivity to protect it from the consequences of uncontrollable stress exposure. ${ }^{21-24}$ Neurobiological mechanisms that have been proposed to convey EE's beneficial effects include alterations in neurotrophin expression, increases in dendritic branching and increased rates of hippocampal neurogenesis. ${ }^{1}$ We tested the hypothesis that adult neurogenesis has a crucial role in the restorative effects of EE on stress-induced behavioral 
manifestations by subjecting both wild-type mice and transgenic mice with conditional blockade of neurogenesis to successive exposures of psychosocial stress followed by either enriched or impoverished home cage conditions. We subsequently tested the ability of $\mathrm{EE}$ to correct the stress-induced depressive-like behavior.

We studied the development of a submissive phenotype in mice exposed to a stressful social conflict (SC) paradigm followed by housing in either an enriched environment (EE) or an impoverished environment (IE) for 3 weeks, and then tested for depressive-like behaviors. One group of mice retained intact adult neurogenesis (Ctrl), whereas another transgenic group had adult neurogenesis conditionally ablated (NG-). We found that Ctrl mice living in EE, as opposed to those living in IE, showed significantly reduced demonstrations of stress-induced submissive, depressive- and anxiety-like behaviors. In contrast NG- mice exposed to EE do not alter their stress-induced behaviors, indicating that the restorative effect of EE critically depend on intact adult neurogenesis. The data showed that the restorative effect of EE critically depends on intact adult neurogenesis.

\section{Materials and methods}

\section{Animals}

We used adult (experimental treatments began at 8 weeks of age and continued to 20 weeks of age) transgenic mice and control littermates from heterozygote (female hGFAPtk) to wildtype (male C57BL/6J) crossings. hGFAPtk transgenic mice were generated as described below and transferred to a C57BL/6J background by $>10$ backcrosses. Before differential housing, animals were housed four per cage in a 12-h light-dark colony room with lights off from 0900 to $2100 \mathrm{~h}$. The procedures described were conducted in accordance with the NIH guidelines and were approved by the NIMH Institutional Animal Care and Use Committee.

\section{Transgenic mouse production}

To generate mice expressing herpes-simplex virus thymidine kinase (HSV-tk) under the control of the human GFAP promoter, the pGFA2-TK2 construct (a generous gift from Dr Michael Brenner, University of Alabama, Birmingham, AL, USA) containing $\sim 2 \mathrm{~kb}$ of the hGFAP promoter fused to the tk gene ${ }^{25}$ was used to generate founder hGFAPtk mice. Transgenic mice were generated by microinjecting $2 \mathrm{pl}$ of a solution of plasmid DNA into the male pronucleus of fertilized oocytes from a mixed C57BL/6J and DBA2 F1 background. Founder mice and offspring were identified by PCR analysis of DNA extracted from tail snips.

\section{Drugs}

Valganciclovir (VGCV, Roche, Indianapolis, IN, USA)— the L-valyl ester of ganciclovir-was administered for up to 12 weeks through the animals' chow at a concentration of $15 \mathrm{mg} \mathrm{kg}^{-1}$ body weight/day. After phosphorylation by HSV-tk, ganciclovir is toxic to proliferating cells in S-phase of mitosis. As control mice do not express HSV-tk, VGCV administration does not suppress proliferation of GFAP-positive cells in these animals. To control for any other effects of the drug, both control and hGFAPtk mice received the same VGCV-containing chow.

\section{Social conflict (SC)}

Repeated social defeat was used to induce a depressive-like phenotype in intruder mice during the SC induction phase. Aggressor CD-1 male mice were single-housed in a large polycarbonate cage $(24.0 \mathrm{~cm} \times 46.0 \mathrm{~cm} \times 15.5 \mathrm{~cm}$, Lab Products, Seaford, DE, USA) for $2-4$ weeks with bedding incompletely refreshed once per week. Male Ctrl and NG- mice were subsequently placed into the resident CD-1 mouse's home cage into which a 1/8-inch thick perforated transparent polycarbonate partition had been placed down the middle to separate the pair. The partition allowed olfactory, visual and auditory communication but prevented tactile contact. Testing commenced after a two-day accommodation period. The partition was removed for $5 \mathrm{~min}^{-1}$ for 14 consecutive days (between 1600 and $1800 \mathrm{~h}$ ) to allow agonistic encounters between the mice. After each interaction period, the partition was replaced with the aggressor male returned to his initial compartment; therefore experimental mice were consistently housed with the same CD-1 mouse. Interaction periods were videotaped under red light and later analyzed by an unbiased observer for aggressive (tail rattles, approaches towards resident/ dominant mouse), submissive (immobility behavior, withdrawals from resident/dominant mouse), and exploratory behaviors. The procedure consistently yielded a subordinate/submissive phenotype in the experimental intruder mice. After 14 interaction periods, experimental intruder mice were randomly divided and placed in either impoverished (IE) or enriched (EE) housing for 21 days.

\section{Impoverished housing (IE)}

Impoverished housing consisted of a $(14.0 \mathrm{~cm} \times$ $35.5 \mathrm{~cm} \times 13.0 \mathrm{~cm}$ ) polycarbonate cage (Tecniplast, Montreal, Canada) with wood chip bedding. To avoid any novel sensory stimulation, bedding was not changed for the entire IE housing period.

\section{Enriched housing (EE)}

Enriched housing consisted of a $(24.5 \mathrm{~cm} \times 40.5 \mathrm{~cm} \times$ $18.5 \mathrm{~cm}$ ) polycarbonate cage (Tecniplast) with wood chip bedding, nesting material, running wheels and numerous polycarbonate and paper tubes of different shapes and sizes (Bio-Serv, Frenchtown, NJ, USA). To minimize stress from novel objects and to maintain a sanitary environment, polycarbonate tubes and wheels were washed with warm water, dried and replaced in the originating cage once every 7 days 
(see Supplementary Figure 3a for comparative cage sizes).

\section{Reintroduction into social conflict (SC)}

Following 21 days of differential housing, experimental mice were reintroduced into SC environment and co-housed with the same CD-1 mouse from the initial induction period for another 14-day period. Similar conditions to the first resident-intruder induction period were used.

\section{Novel cage test}

During the dark cycle of the second SC period, experimental animals were placed in a fresh polycarbonate mouse cage $(14.0 \mathrm{~cm} \times 35.5 \mathrm{~cm} \times 13.0 \mathrm{~cm})$ (Tecniplast) and allowed to freely explore. Explorations were videotaped from a side view for $1 \mathrm{~h}$. Distance moved was analyzed from the video files using HomeCageScan Software (Cleversystems, Leesburg, VA, USA).

\section{Saccharine preference test}

Mice were subjected to a 2-bottle choice saccharine (50 $\mathrm{mg} \mathrm{l}^{-1}$ ) preference test. Testing was carried out over 3 days in the home cage. Each mouse was given simultaneous access to two dual ball sipper-top bottles (AnaCare, Potomac, MD, USA): one with purified Milli-Q water and one containing a $50 \mathrm{mg} \mathrm{l}^{-1}$ saccharine (Sigma Aldrich, St Louis, MO, USA) solution ad libitum. The amounts consumed over the whole 3-day period were measured and the preference for the saccharine solution was calculated according to the formula: Percentage of preferen$\mathrm{ce}=(($ saccharine solution intake/total intake $) \times$ $100 \%)$.

\section{Light-dark box test}

The light-dark box test was conducted using a Plexiglas box $(50 \mathrm{~cm} \times 25 \mathrm{~cm}$ with $30 \mathrm{~cm}$ walls $)$ consisting of a dark (1/3 of the box) and a transparent (2/3 of the box) compartment that was illuminated to approximately 40 lux. An open door divided the compartments. Each mouse was placed in the light compartment and allowed to freely move within the compartments for $10 \mathrm{~min}$. The time spent in the dark compartment was measured as an indicator for anxiety-like behavior.

\section{Social-interaction and dominant-interaction tests}

Mice were placed into an open field containing 2 upside-down wire mesh pencil cups that served both as novel objects and container for another mouse. In the social interaction test, the mouse placed in the cup was an unfamiliar mouse of same genetic background (C57BL/6J) and age. In the dominant interaction test (performed 1 day after the social interaction test), the cup contained the same aggressor that the animal had lived with under the SC conditions. Mice were placed in the middle of the open field and allowed to explore for $1 \mathrm{~h}$. Sessions were videotaped and later analyzed using automated behavioral tracking software (TopScan/ObjectScan, Cleversystems, Leesburg, VA, USA). The software reliably scores the frequency and duration of sniffing on either object or mouse through identification of the animals shape including nose, body and tail. Interaction ratios were calculated using the interaction quotient (interaction duration on mouse/interaction duration on empty cup).

\section{Analysis of neurogenesis by immunohistochemistry}

To analyze neurogenesis, BrdU $\left((+)-5^{\prime}\right.$ bromo2'deoxyuridine; 97\%; Sigma-Aldrich, St Louis, MO, USA) immunohistochemistry was used. Different BrdU injection schedules were used depending on the objective of the experiment. BrdU was injected i.p. at a concentration of $200 \mathrm{mg} \mathrm{kg}^{-1}$ body weight. This concentration was previously determined to be the lowest dose labeling a maximum number of cells (data not shown). To examine the survival of adultborn neurons in different housing conditions, mice were administered $\mathrm{BrdU}$ (200 $\mathrm{mg} \mathrm{kg}^{-1}$ i.p.) once per day for five consecutive days before differential IE, EE or SC housing conditions. Mice were killed 4 weeks after the last injection. To evaluate proliferation in different housing conditions BrdU was injected once and the animals were perfused $3 \mathrm{~h}$ later. To evaluate the proliferation and short-term survival in different areas of the brain of GFAPtk transgenic mice (Figure 1f) a high number of BrdU injections (5 consecutive days, twice daily) was chosen to label most cells including progenitor cells with a low proliferative rate. Tissue was collected $24 \mathrm{~h}$ after the last injection. Brains were cut into coronal sections $(40 \mu \mathrm{m})$ and serially collected in 12 wells of a 24-well plate containing PBS, $\mathrm{pH}$ 7.4. Free-floating sections from one well were washed for $15 \mathrm{~min}$ in PBS, $\mathrm{pH} 7.4$ containing $0.5 \%$ Tween 20 and subsequently incubated for $30 \mathrm{~min}$ at RT in blocking solution (PBS, pH $7.4,0.5 \%$ Tween $20,3 \%$ normal donkey serum). Following blocking, sections were incubated overnight at $4{ }^{\circ} \mathrm{C}$ with the primary antibody (Rat AntiBrdU Clone BU1/75, 1:500, Accurate, Westbury, NY, USA). Sections were washed for $15 \mathrm{~min}$ followed by incubation for $2 \mathrm{~h}$ at RT with secondary antibodies (Alexa-488 donkey anti-rat IgG, 1:250, Invitrogen Corporation, Carlsbad, CA, USA). Other primary antibodies used in this study were used to detect doublecortin (DCX) (anti-DCX, Santa Cruz Biotechnology, Santa Cruz, CA, USA), Iba-1 (anti-Iba-1, WAKO Chemicals, Richmond, VA, USA) and GFAP (anti-GFAP, Sigma-Aldrich, St Louis, MO, USA). Sections were counterstained using DAPI (Sigma), mounted on glass slides, and coverslipped with aqueous mounting medium (Immu-Mount, ThermoFisher Scientific, Waltham, MA, USA). Fluorescently immunolabeled sections were analyzed on an Eclipse 800 fluorescent microscope (Nikon Instruments). Confocal photomicrographs of the DG of Ctrl and NG- animals were generated on a Zeiss Meta confocal microscope (Model LSM 510; Carl Zeiss MicroImaging, Thornwood, NY, USA). 

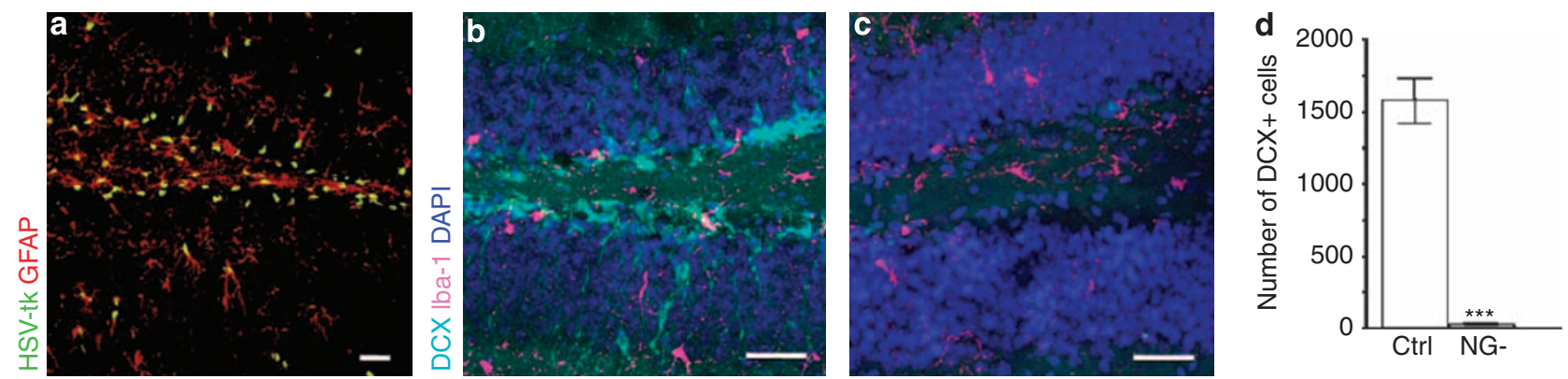

e
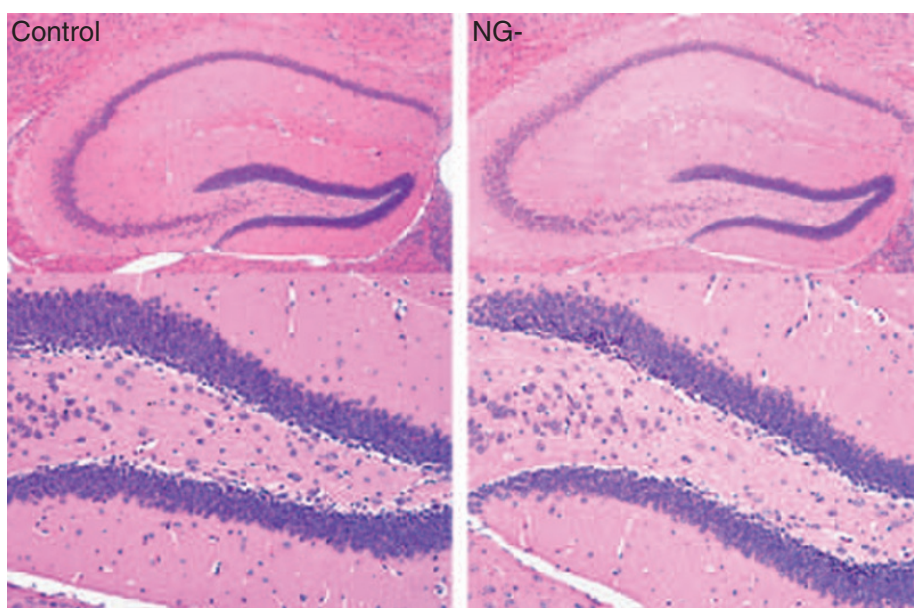

Figure 1 (a) Immunohistochemistry against HSV-tk and GFAP in the hippocampal dentate gyrus (DG) of adult hGFAPtk mice reveals HSV-tk expression in GFAP ${ }^{+}$cells. (b-d) The DG of control mice contains many doublecortin (DCX) positive neuroblasts (aqua) while these cells are virtually abolished $\left(F_{8,8}=428.2\right.$, Student's $t$-test $\left.P<0.0001\right)$ in hGFAPtk mice treated with VGCV for 3 weeks (NG-, $26.67 \pm 7.688, n=9$, gray bar; Controls, $1577 \pm 159.1, n=9$, white bar) . Staining for Iba1 (purple) reveals no increase in activated microglia. Scale bars $=30 \mu \mathrm{m}$. (e) Hematoxylin and Eosin (H\&E) staining shows no indication of inflammation in the hippocampus of NG- mice treated with VGCV for $>8$ weeks. (f) Analysis of cell proliferation and short-term survival in the adult CNS of Ctrl and NG- mice. After $>8$ weeks of VGCV treatment mice were injected twice daily for 5 days with BrdU (200 mg kg-1 body weight) and perfused $24 \mathrm{~h}$ after the last injection. BrdU ${ }^{+}$cells were counted in the hippocampal DG and Cornu Ammonis regions (CA3 and CA1), in the hypothalamus (Hy) including the paraventricular nucleus (PVN), as well as in medio-prefrontal cortical regions (mPFCx), motor cortex ( $\mathrm{mCx})$ and the subventricular region of the lateral ventricles (SVZ). A decrease in BrdU ${ }^{+}$cells was only detected in the DG $\left(\mathrm{F}_{3,5}=12.27\right.$, Student's $t$-test $\left.P=0.0155\right)$ and the SVZ $\left(F_{3,5}=1.691\right.$, Student's $t$-test $\left.P<0.001\right)$. (Control, $n=4$, white bars, NG- $n=6$, black bars) Note that NG- mice still show a high rate of cell proliferation in the DG and SVZ. This is most likely due to the generally high proliferative capacity in these regions including cell types of non-neuronal lineages that do not undergo differentiation into DCX ${ }^{+}$positive neuroblasts such as microglial cells, endothelial cells as well as oligodendrocyte progenitor cells.

\section{Statistical analysis}

For the social conflict sessions, behavioral data gathered during the induction period were analyzed by a paired samples $t$-test, and behavioral data gathered during the re-exposure period were analyzed by repeated measures ANOVA with group as between factor (EE/Ctrl, EE/NG-, IE/Ctrl, IE/NG-) and day as within factor (14 levels: day 1-day 14). If significant main effects or interactions were confirmed, individual one-way ANOVAs and post hoc tests were used to further assess the nature of the effect (Supplementary Table 2). One-way ANOVA was also used for experiments that compared effects of treatment. Statistics were analyzed using software packages from SPSS 16.0 for Macintosh, and significance was determined at $P<0.05$. Data are expressed as mean \pm s.e.m.

\section{Results}

\section{Conditional ablation of adult neurogenesis}

Expression of HSV-tk in GFAP-positive cells was confirmed using immunohistochemistry against HSV-tk and GFAP (Figure 1a). HSV-tk phosphorylates the prodrug VGCV into toxic metabolites that specifically kill actively dividing, DNA synthesizing cells. Dividing neural progenitors in the SGZ of the hippocampal DG express $\mathrm{GFAP}^{26}$ and hence, in hGFAPtk mice, these cells are ablated by administration of VGCV. Following 3 weeks of VGCV adminis- 
tration, doublecortin (DCX)-positive neuroblasts cannot be detected in the hippocampus of hGFAPtk mice (NG- mice) (Figure 1b, c and d). No signs of inflammation due to HSV-tk-induced death of GFAP-positive progenitors were observed, as determined by expression of Iba1, a marker of activated microglia (Figure $1 \mathrm{~b}$ and $\mathrm{c}$ ) or by standard pathological hematoxylin and eosin (H\&E) staining (Figure 1e).

To determine the brain regions in which proliferation is affected in hGFAPtk mice, we injected Ctrl and NG- mice with BrdU twice a day for 5 consecutive days. BrdU cell counts revealed that proliferation was selectively affected in the two known neurogenic regions of the brain-the subventricular zone (SVZ) of the lateral ventricles and the SGZ of the hippocampal DG. No changes in cell proliferation were observed in other regions of the brain including the hypothalamus, prefrontal cortex, motor cortex and the hippocampal CA regions (Figure 1f).

No unwanted side effects that could compromise the general health of the animal were apparent in VGCV-treated hGFAPtk mice. No changes were detected in NG- mice in any parameters of a comprehensive physical examination that included physical characteristics, general behavioral observations, sensorimotor reflexes and motor responses
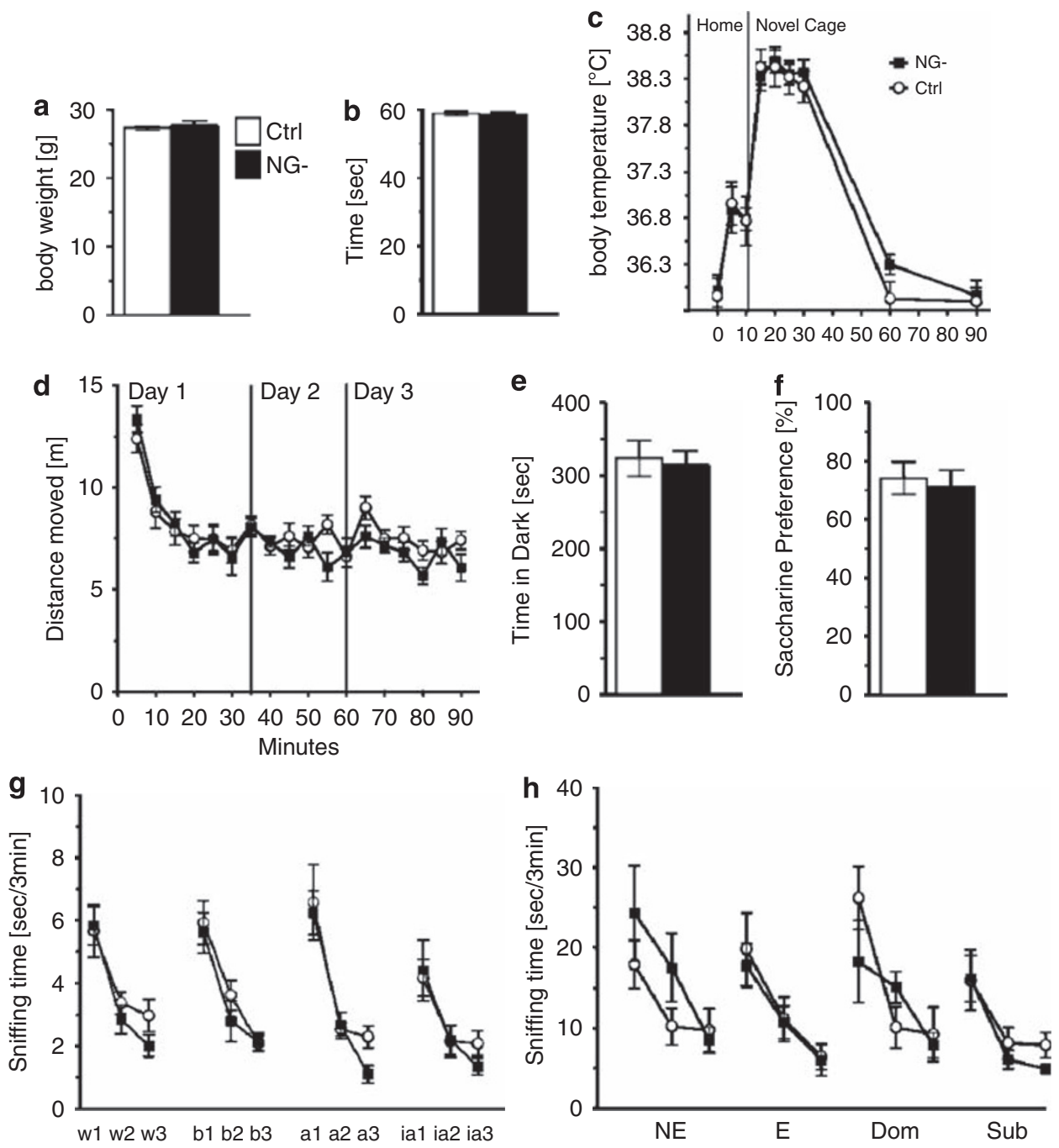

Figure 2 Control and NG- mice show no differences in baseline physiology, no changes in baseline behavioral tests for locomotion, anxiety and depressive-like behavior, and no difference in olfactory habituation/dishabituation. hGFAPtk mice treated for 8 weeks with VGCV (NG-) do not show any differences in body weights (a), motor strength in the wire hang test (b) or novel cage stress-induced hyperthermia (c). NG- mice do not differ from controls in activity in or habituation to an open field (d), show no differences in anxiety or exploration in the open field or light-dark box test (d and e) and have normal saccharine preference (f). NG- mice do not differ from controls in an olfactory habituation dishabituation test for standard odors (g) nor for odors carrying a social connotation (h). Cotton tips dipped in different odors; water (w1-3), banana (b1-3), almond (a1-3) and imitation almond (ia1-3) were introduced to the animals homecage three consecutive times for 3 min. Both NG- and control mice spend the same amount of time sniffing and habituating to the different odors (g). Similarly, no difference was found between control and NG- mice sniffing and habituating to cotton tips dipped in urine from female animals in non-estrous (NE) or estrous (E) phase of their estrous cycle as well as urine from dominant (Dom) CD-1 animals and subordinate (Sub) C57Bl/6J animals (h). 
(Supplementary Table 1). An extensive pathological examination of hGFAPtk mice after prolonged ( $>8$ weeks) VGCV treatment did not reveal any functionally significant effects of the antiviral drug treatment (pathology reports are provided as Supplementary Material). No differences in body weight, motor strength or stress-induced hyperthermia were detected in VGCV-treated hGFAPtk mice lacking neurogenesis even after prolonged VGCV treatment ( $>8$ weeks) (Figure 2a, b and c). Baseline behavior of NG- mice compared with controls differed neither in measurements of locomotion and exploration, nor in anxiety and hedonic drive (Figure 2d, e and f).

Although proliferation of progenitor cells in the SVZ, a site generating olfactory bulb cells, is affected in the VGCV-treated transgenic mice (SVZ-Figure 1f), NG- mice discriminate between and habituate normally to both standard odors as well as those carrying different social connotations (Figure $2 g$ and $h$ ).

Published reports have indicated that a GFAPtk mouse line, in which HSV-tk is driven by the mouse GFAP promoter, expresses tk in intestinal glial tissue and that ganciclovir treatment leads to a severe Morbus Crohn-like inflammatory phenotype. ${ }^{27}$ In our mice, no functionally significant peripheral actions of the construct were present, as determined by the aforementioned behavioral and pathological exams.

\section{Enriched environment and chronic social defeat differentially affect long-term survival of adult born neurons}

To examine the effects of the different living conditions used in our experiments (EE, IE and SC) on adult hippocampal neurogenesis, we conducted BrdU labeling and stereological cell counting to quantify the 4-week survival of newly born cells in the DG (Figure 3a-c). As expected, ${ }^{11} 3$ weeks of EE significantly increased the extent of cell survival compared with both the IE and SC groups (Figure 3c). We also examined the effects of the different living conditions on proliferation of newborn neurons in the DG by giving a single injection of BrdU $3 \mathrm{~h}$ before perfusion. Two weeks of SC or 3 weeks of either EE or IE had no effect on the rate of proliferation in the DG (Figure 3d and e).

\section{Repeated social defeat in the SC paradigm leads} to a submissive phenotype

Male mice develop social hierarchies based on agonistic (aggressive) encounters. Although all participants initially display overt aggressive behavior, winners and losers emerge after repeated interactions. In the SC paradigm (see Figure 4a for an experimental timeline), a stable dominant-subordinate hierarchy formed over time, and repeated defeats rendered the test mice chronically submissive. The extent and form of agonistic interaction during the SC induction period was similar for the Ctrl and NG- groups (Figure 4b-d and Supplementary Figure 1). Initially, both Ctrl and NG- mice displayed an equally strong aggressive phenotype directed towards the CD-1 a
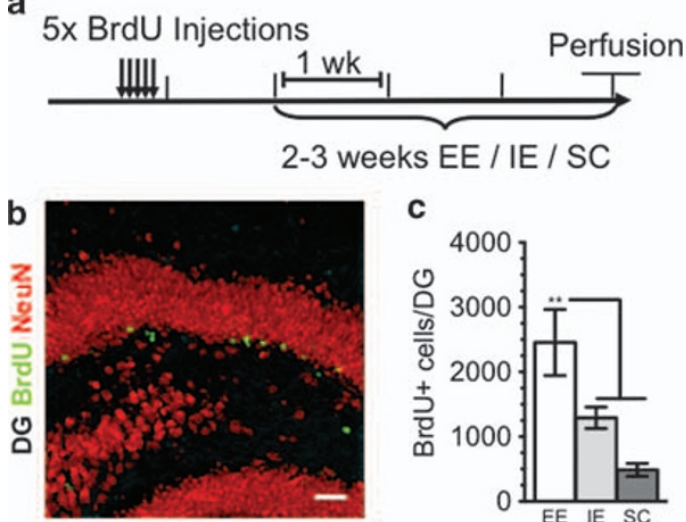

C
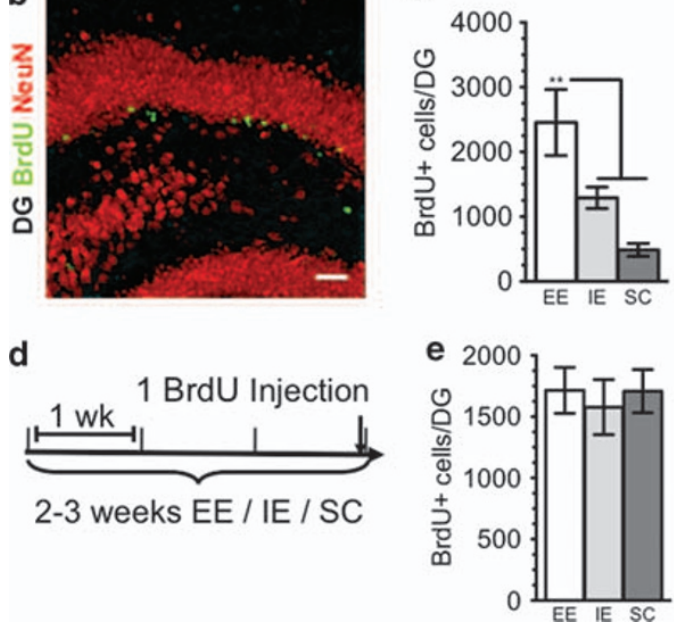

Figure 3 (a) Timeline shows BrdU injections for studying survival of newborn neurons under different housing conditions. Animals were injected with BrdU $\left(200 \mathrm{mg} \mathrm{kg}^{-1}\right)$ $1 \times$ per day for 5 days, 1 week before being placed in either an enriched environment (EE) or an impoverished environment (IE) for 3 weeks before perfusion. In the social conflict (SC) condition, animals were injected $1 \times$ per day for 5 days, 2 weeks before being placed in SC for 2 weeks before perfusion. (b) Newborn neurons were analyzed using double labeling for BrdU (green) and the neuronal marker, NeuN (red). Almost all BrdU-labeled cells also expressed NeuN. (c) Animals living in EE displayed significantly increased survival of newborn neurons as compared with either animals in IE or SC (EE, $2838 \pm 562.8, n=6$, white bars; IE, $1291 \pm 164.8, n=5$, gray bars; SC, $572.0 \pm 45.18$, $n=6$, dark gray bars; $F_{2,14}=10.98, P=0.0014$; Tukey-Kramer post hoc test for EE vs IE $P<0.05$, for EE vs SC $P<0.01$ ). (d) Timeline shows BrdU injections for studying proliferation of newborn neurons under differential housing conditions. Animals were given a single injection of BrdU $3 \mathrm{~h}$ before being euthanized after having lived in either EE, IE or SC housing conditions for $2-3$ weeks (EE, $1715 \pm 530.5$, $n=8$, white bars; IE, $1578 \pm 634.2, n=8$, gray bars; SC, $1709.0 \pm 499.2, n=8$, dark gray bars).

mouse as shown by elevated approaches towards the CD-1 mouse (Figure 4c), brief time spent immobile (Figure 4d), and high number of tail rattles (Supplementary Figure 1c). Aggressive behaviors in both groups rapidly declined and were absent by the seventh day of the induction period as social defeats accumulated. Immobility behavior offers a striking example of the powerful effect of SC on behavior. On days 1-3, mice in both groups spent less than $50 \mathrm{~s}$ of the 5 -min encounter immobile (Figure $4 \mathrm{~d}$ ). However, immobility time increased to $\sim 100 \mathrm{~s}$ on days $6-8$, and by day 11 , mice in both groups spent the majority of the session immobile. Both groups also 
showed similar decreases in latency to conflict, number of explorations and relatively constant numbers of conflicts, and withdrawals across the sessions (Supplementary Figure 1).

Enriched environment aids recovery from social conflict-induced submission, which is dependent on adult neurogenesis

After 14 days, defeated Ctrl and NG- intruder mice were removed from the SC condition and placed into either the EE or IE condition for 3 weeks. At the end of this period, both groups were re-exposed to the SC condition. Behavioral measures were again scored during the daily interaction period for the 2 weeks of SC re-exposure (Figure $4 \mathrm{e}-\mathrm{g}$ and Supplementary Figure 2).

Exposure of defeated animals to EE versus IE had a strong effect on behavior during the SC re-exposure period. Whereas IE-exposed mice displayed a strong submissive phenotype similar to behavior observed at the end of the SC induction, EE-exposed mice were more aggressive, displaying behavior similar to that observed at the beginning of the SC induction. Upon initial re-exposure to SC, both EE groups (Ctrl and NG-) won more conflicts when compared with both IE groups (Ctrl and NG-) (Figure 4e). However, when comparing the EE/Ctrl to EE/NG- groups, Ctrl mice won significantly more social conflicts during days 1-3 as compared with NG- mice. Strikingly, by the fourth conflict session, any similarity between EE/Ctrl and EE/NG- mice had disappeared. Remarkably, only the EE/Ctrl mice displayed continuously high levels of conflict victories throughout the SC re-exposure period, indicating that EE had an enduring beneficial effect in Ctrl animals. Accordingly, after the initial three sessions the EE/Ctrl group displayed more approaches (Figure 4f) and spent less time immobile (Figure 4g) as compared with all other groups.

In marked contrast to the EE/Ctrl group's behavioral recovery, the EE/NG- group fared as poorly as both IE groups despite living under EE conditions. When compared with EE/Ctrl from the third conflict session on, the EE/NG- group won fewer conflicts (Figure 4e), spent more time immobile (Figure 4g), and showed fewer aggressive behaviors including approaches towards the resident mouse (Figure 4f) and tail rattles (Supplementary Figure 2c). During the early conflict sessions, EE/NG- mice showed a modest increase in exploratory behavior compared with both IE groups. However, this effect disappeared after the third conflict session (Supplementary Figure 2d). Results for repeated measures ANOVA are shown in Supplementary Table 2.

\section{Affective behaviors}

During the final 7 days of the SC re-exposure period, we conducted a battery of behavioral tests to further elucidate the affective phenotype of Ctrl and NGmice after either $\mathrm{SC} \rightarrow \mathrm{EE}$ or $\mathrm{SC} \rightarrow \mathrm{IE}$. The same order of tests: exploration in a novel cage, preference for a sweet solution, anxiety, social preference and preference for the intruder/aggressor, was conducted for each group to assay affective behaviors (Figure 4a).

Exploratory activity. To assess novelty-induced exploratory activity, we measured locomotor activity

Figure 4 Diagram depicting experimental groups and study design (a). An initial cohort consisting of control and NG- mice were first housed for 14 days under social conflict (SC) conditions during which time aggressive encounters were scored for 5 min day ${ }^{-1}$. Following SC induction, all animals were removed from the SC living condition and divided into four groups: 1. Control animals living under EE conditions, 2. NG- animals living under EE conditions, 3. Control animals living under IE conditions and 4. NG- animals living under IE conditions. Following 3 weeks of either the EE or IE condition, all groups were re-exposed to SC for 14 days during which aggressive encounters were scored for 5 min day $^{-1}$. In addition, all groups underwent a behavioral battery during the final week of the SC condition (a). SC induction period (b-d): Chronic psychosocial stress and daily social defeat induce subordinate/submissive phenotype in Ctrl and NG- mice. The induction of submissive behavior was determined by; (b) percentage of conflicts won, (c) the number of approaches by the intruder mouse towards the resident dominant CD-1 mouse and (d) time spent immobile. As NG- $(n=22)$ and control $(n=22)$ mice accumulated social conflict defeats, aggressive behaviors declined and immobility increased. Ctrl and NG- mice showed no significant differences in the development of submissive behavior. SC Re-exposure (e-g): In previously submissive male mice, hippocampal neurogenesis and environmental enrichment opposes the development of subordinate phenotype during re-exposure to chronic psychosocial stress. Compared with all other groups, EE Ctrl mice showed; (d) a significant increase in percentage of conflicts won (significant effect of group $\left[\mathrm{F}_{3,41}=73.66 ; P<0.001\right]$, day $\left[\mathrm{F}_{13,29}=2.581 ; P<0.02\right]$ and a significant interaction between group $\times$ day $\left[\mathrm{F}_{13,29}=2.053 ; P<0.01\right]$, $(\mathbf{e})$ a significant increase in approaches towards resident $\mathrm{CD}-1$ mouse (significant effect of group $\left[\mathrm{F}_{3,41}=22.54 ; P<0.001\right]$, and (f) a significant decrease in time spent immobile during conflict sessions (significant effect of group $\left[\mathrm{F}_{3,41}=25.302 ; P<0.001\right]$, day $\left[\mathrm{F}_{13,29}=2.966 ; P<0.01\right]$. ${ }^{*}$ indicates significant difference $(P<0.05)$ between EE Ctrl and all other treatment groups. **indicates significant difference $(P<0.05)$ between EE Ctrl and IE Ctrl/IE NG- groups. ${ }^{* * *}$ indicates significant difference $(P<0.05)$ between Ctrl and NG- groups. $N=11$ per group. Data are expressed as mean \pm s.e.m. (h) EE exposed groups show higher levels of exploration in the first 15 min in a novel environment compared with all other treatment groups (significant effect of treatment: $\mathrm{F}_{3,19}=5.268, P<0.01$ ). $n=6$ per group. (i) Only animals living in EE with intact neurogenesis showed preference for saccharine (values above 50\% [horizontal line] indicate preference for saccharine solution above chance) (significant effect of treatment: $\mathrm{F}_{3,38}=3.539, P=0.0235$ ). $n=10-11$ per group. (j) In the light-dark box test, animals that had lived in EE with intact neurogenesis spend less time in the dark compartment compared with all other groups (significant effect of treatment: $\mathrm{F}_{3,39}=12.41, P<0.0001$ ). $n=10-11$ per group. Results are expressed as mean \pm s.e.m. *indicates significantly different $(P<0.05)$ from all other treatment groups. One-way ANOVA followed by Newman Keuls post hoc test. 
for $1 \mathrm{~h}$ in response to novel cage introduction. Mice in all groups showed a gradual decrease in exploratory activity during the session, indicating that they habituated normally to the novel environment (data not shown). However, in the first $15 \mathrm{~min}$ of the session, Ctrl/EE mice displayed significantly higher exploratory activity as compared with all other groups (Figure 4h).

Saccharine preference. We used the saccharin preference test as a read-out for hedonic behavior. Mice normally prefer a sweetened solution (that is, saccharin) to water, and this preference is reduced in chronically stressed mice. Remarkably, only the Ctrl/EE group showed the expected preference for saccharin $(\sim 70 \%)$, while all other groups showed preference at chance levels ( $\sim 50 \%)$ (Figure $4 \mathrm{i})$.

Anxiety. To measure anxiety-like behavior, we used the Light-Dark Box test, a commonly used behavioral test in which mice are allowed to explore a box consisting of a light and a dark compartment. The amount of time spent in the dark and the frequency of visits to the light is a frequently used measure of anxiety-like behavior in mice. We found that Ctrl/EE mice showed decreased levels of anxiety-like behavior as compared with all other groups (Figure $4 \mathrm{j}$ ).

Social and agonistic preference. To assess baseline social interaction, we measured the time the experimental mouse spent interacting with a wire
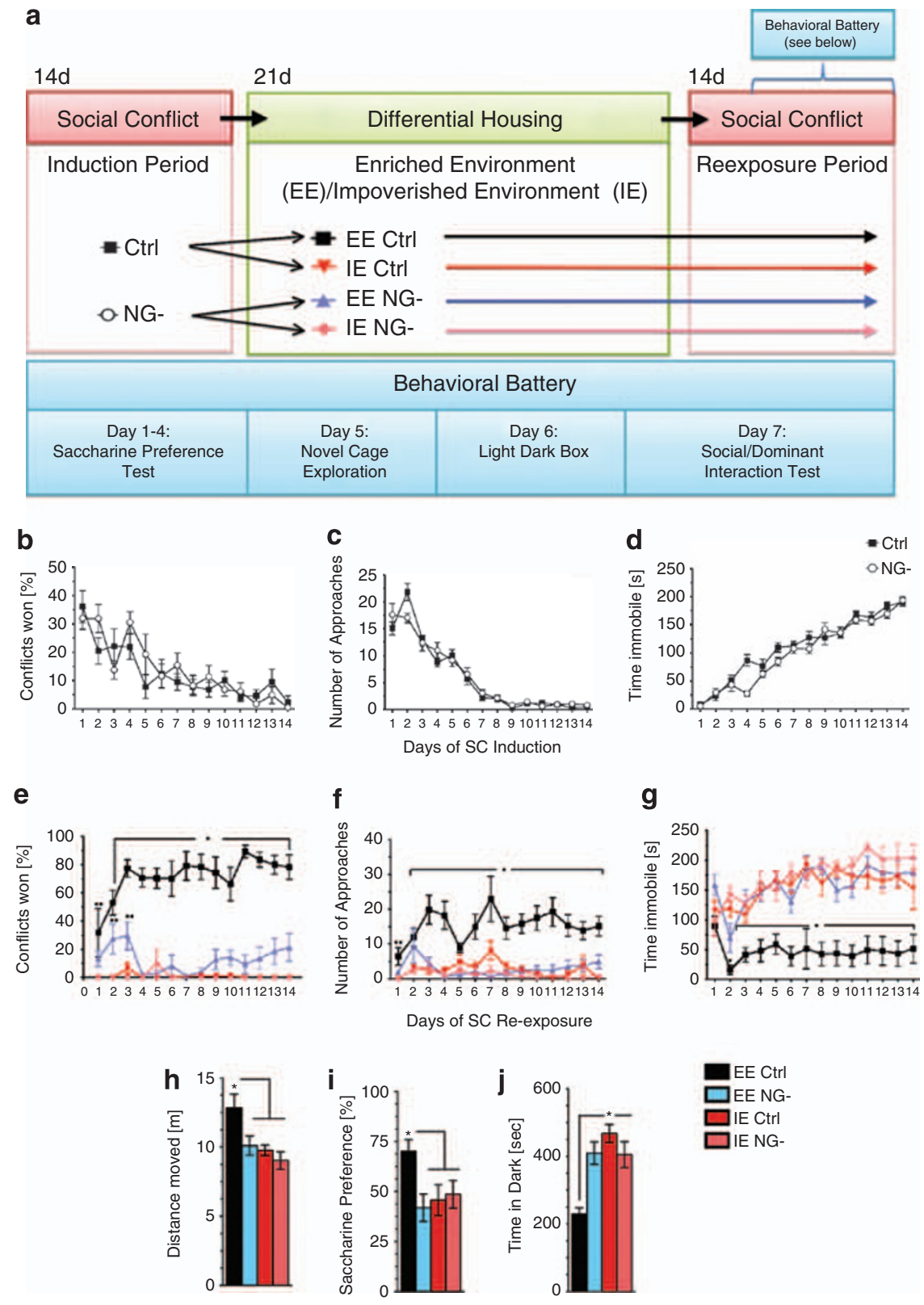

EE Ctrl

$\square$ EE NG

$\square$ IENG 

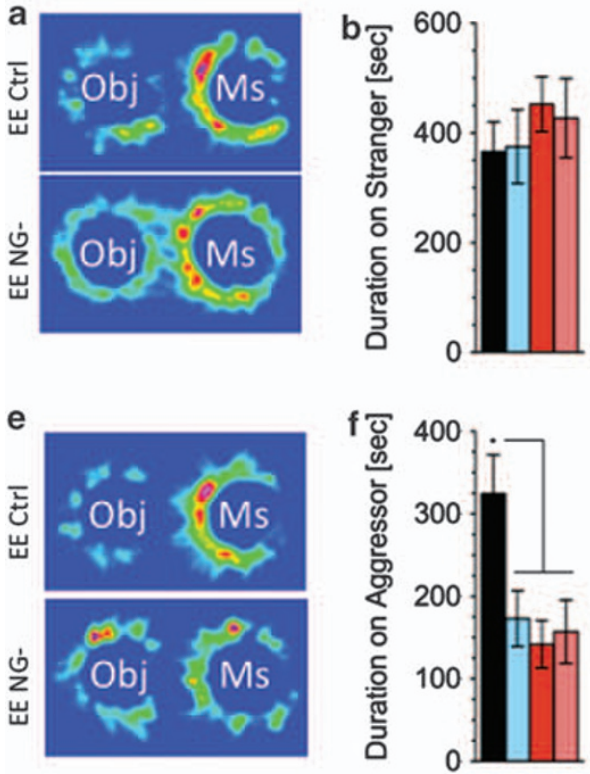
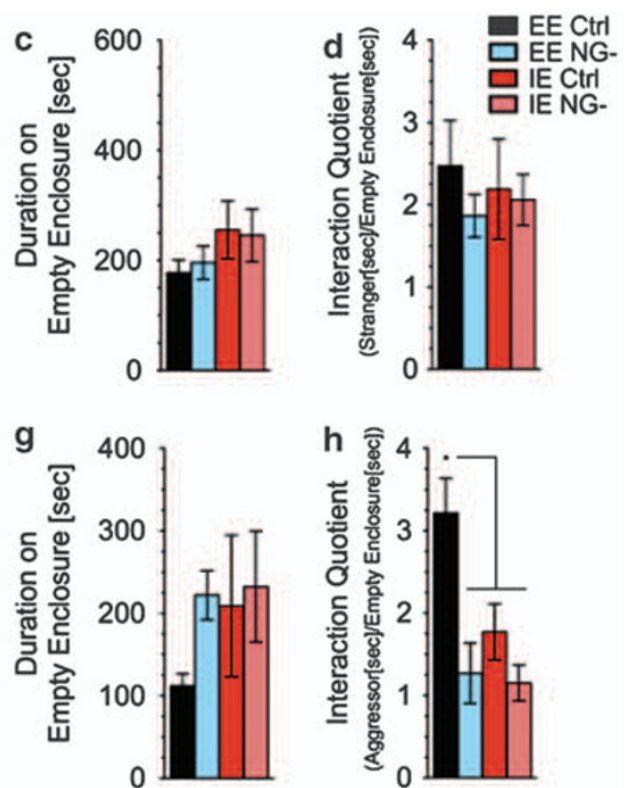

Figure 5 Social exploration of an aggressor mouse is restored by EE only when neurogenesis is intact. Mice were tested in two social interaction tests. In the first, mice were allowed to explore an open field containing two wire cups, one empty and one containing an unfamiliar mouse. In the second test the unfamiliar mouse was replaced with the known aggressor from the SC living conditions. (a-d) No difference in behavior was observed between treatment groups when mice were exposed to an unfamiliar mouse. All groups preferred (Interaction Quotient Duration on Mouse [s]/Duration on Object [s] $\cong 2$ ) interacting with an unfamiliar mouse compared with interacting with an inanimate object. (e-h) However, when the aggressor was presented EE NG- mice as well as both IE groups spend significantly less time with the mouse than the EE Ctrl group (significant effect of treatment; $\mathrm{F}_{3,41}=5.097, P<0.005$, in f) The interaction quotient was also significantly higher in EE $\mathrm{Ctrl}$ as compared with all other groups (significant effect of treatment; $\mathrm{F}_{3,41}=7.553, P<0.001$, in $\mathbf{h}$ ). Results are expressed as mean \pm s.e.m. $n=11$; *indicates significantly different $(P<0.05)$ from all other treatment groups. One-way ANOVA followed by Tukey-Kramer post hoc test.

mesh enclosure containing a similarly aged and sized C57BL/6J stranger mouse as opposed to an empty wire mesh enclosure (Supplementary Figure 4c). Mice can see, smell and explore the animal under the enclosure, but agonistic encounters are prevented (Supplementary Figure 4c). The experimental mouse is placed in the center of the arena and the time spent interacting with an enclosure containing a naive/ stranger mouse or with an empty enclosure is scored. No differences between the four groups were observed in the time spent interacting with either the strangercontaining enclosure (Figure 5a and b) or with the empty enclosure (Figure 5a and c). Mice in all groups showed an interaction quotient (duration on stranger/ duration on empty enclosure) at $\sim 2$ (Figure $5 \mathrm{~d}$ ), indicating a preference for the stranger-containing enclosure as opposed to the empty enclosure.

We next used a similar paradigm to examine agonistic social interaction. One wire mesh enclosure remained empty whereas the other contained the dominant aggressor mouse with which the experimental mouse had been paired during the SC condition (Supplementary Figure 4d). Time spent interacting with the aggressor-containing enclosure (Figure 5e and f) as opposed to the empty enclosure (Figure 5e and g) was scored. When compared with all other groups, Ctrl/EE mice spent more time interacting with the aggressor-containing enclosure (Figure 5f) and displayed a significantly higher interaction quotient (duration on aggressor/duration on empty enclosure) (Figure 5h), indicating that they strongly preferred the aggressor-containing enclosure.

\section{Discussion}

Our data show that EE can ameliorate submissive and depressive-like behaviors adopted by male mice in response to chronic psychosocial stress. Exposing subordinate wild-type animals to EE after SC (SC $\rightarrow$ EE) results in increased aggressive displays towards the dominant animal. Compared with $\mathrm{SC} \rightarrow$ $\mathrm{IE}, \mathrm{SC} \rightarrow \mathrm{EE}$ mice display normal hedonic drive, decreased anxiety-like behaviors and increased interaction with the SC partner, essentially restoring the animals' behavior to their pre-stress patterns. Importantly, our data show that EE's restorative effects depend on intact adult neurogenesis. Induction of the SC-induced submissive phenotype is similar between Ctrl and NG- mice. However, NGanimals neither reap the benefit of $\mathrm{EE}$ in extinguishing the SC-induced submissive phenotype nor realize EE's anti-depressive and anxiolytic effects.

We used daily social defeat in combination with chronic non-tactile exposure to induce a subordinate, depressive-like phenotype. Numerous models, including chronic unpredictable mild stress and 
chronic restraint stress, have been developed to induce depressive-like behaviors in rodents. However, these paradigms lack important psychosocial components inherent to human depression. Because stressful life events are often social in nature, ethologically relevant SC animal models may be particularly useful in understanding how differential social experiences lead to neurobiological changes that can influence behavior. Although both participants in an agonistic encounter experience social stress, its consequence is different for winners versus losers, with the social stress experienced by subordinates leading to increases in social withdrawal as well as depressive- and anxiety-like behaviors. In rodents, chronic antidepressant treatment increases aggression, resulting in elevated hierarchical status. ${ }^{28}$ In our study, $\mathrm{SC} \rightarrow \mathrm{IE}$ mice retain their submissive behavior upon SC re-exposure, suggesting an inability to cope with the previous adverse experience. In contrast, $\mathrm{SC} \rightarrow \mathrm{EE}$ mice showed an increase in aggressive behavior upon SC re-exposure, suggesting that $\mathrm{EE}$ promotes the acquisition of adaptive coping strategies.

In humans, physical exercise and positive psychosocial activities can improve cognitive function, reduce depressive symptoms and increase stress resiliency. ${ }^{29}$ In animals, exercise and complex environments exert profound influences on brain structure and chemistry as well as on cognitive and emotionally relevant behavioral measures. ${ }^{30-32}$ It has been suggested that EE insulates the individual from the adverse effects of uncontrollable stress exposure, tempering its emotional reactivity. ${ }^{14,21}$ Animals living in EE show upregulation of hippocampal glucocorticoid receptor (GR) mRNA expression and increased glucocorticoid sensitivity. ${ }^{23,33}$ Accordingly, enriched animals show blunted hypothalamic-pituitary-adrenal (HPA) axis responses to mild stressors. ${ }^{34}$ Moreover, exercise has been shown to enhance habituation of the HPA axis to repeated stressor exposure. ${ }^{35}$ These reports suggest that EE renders the HPA axis more adaptive, resulting in decreased emotional reactivity and increased emotional stability.

The hippocampus provides powerful inhibitory control over the HPA axis, and the DG in particular has been shown to strongly coordinate release of corticotropin-releasing hormone in the hypothalamic paraventricular nucleus. ${ }^{36}$ We recently reported that loss of adult neurogenesis alters the HPA-axis response to mild stress, ${ }^{37}$ suggesting a role for adult neurogenesis in mediation of HPA-axis activity by the hippocampus. The significance of HPA-axis modulation in depression is underscored by reports that successful antidepressant treatment is associated with resolution of impairment in HPA-axis negative feedback. ${ }^{38,39}$ Our data show that the ability of animals to recover from the SC-induced submissive phenotype is critically dependent on intact neurogenesis. Given previous evidence that the HPA-axis is altered by EE exposure and that neurogenesis modulates the HPA-axis stress response, it is plausible that the antidepressant effects of EE in behavioral recovery from SC are mediated via regulation of the HPA axis by neurogenesis.

Two fundamental lines of research have pointed to a link between hippocampal neurogenesis and depression. First, stress, an important etiological factor in the pathogenesis of depression, decreases hippocampal neurogenesis. Second, many antidepressant therapies, including electroconvulsive therapy, exercise and antidepressant drugs, enhance hippocampal neurogenesis. ${ }^{40-43}$ Moreover, pharmacological therapies have a delayed onset of efficacy that parallels the protracted time scale of increased neurogenesis. The functional significance of antidepressant-induced enhancement of neurogenesis was shown in rodents with the report that ablation of cell proliferation by hippocampal irradiation blocked specific behavioral effects of antidepressants. . $^{9,44}$

It remains unclear whether impaired neurogenesis and development of depression per se are causally related. Adult neurogenesis and depression are not well correlated in several rodent models of depression, and ablating neurogenesis does not appear to affect baseline anxiety- and depressive-like behaviors ${ }^{10,45}$ Our results support these data, showing that loss of neurogenesis does not directly lead to depressive- or anxiety-like behaviors (Figure 2e and f). We do, however, uncover a role for neurogenesis in mediating the beneficial effects of EE in recovery from a depressive-like state. In both Ctrl and NG- animals, SC induction led to a submissive phenotype, but only Ctrl animals were able to take advantage of EE for recovery. A recent report by Meshi et al. ${ }^{46}$ showed that hippocampal neurogenesis was not required for baseline EE-induced improvements in spatial learning and anxiety-like behavior. These previous results coupled with our data lend support to the idea that hippocampal neurogenesis does not mediate baseline improvements in behaviors, but it may be specifically important in the remediation of impaired behaviors.

In addition to the possible effects of $\mathrm{EE}$ and neurogenesis on stress responsiveness via the HPA axis, new neurons may be influencing contextspecific memory formation, which could contribute to EE's ability to remediate behavioral impairments in our SC model. Hippocampal network models suggest that increased DG cell turnover can enhance encoding of new memories, whereas simultaneously degrading old memory recall. ${ }^{47,48}$ Supporting this idea, it has been proposed that the DG and specifically its newly born neurons, may have a unique role in 'pattern separation', enabling the animal to amplify differences between old and new representations of an experience. ${ }^{49-51}$ Their physiological properties render adult-born DG neurons more sensitive to successive exposures to the same environment, ${ }^{52}$ which could provide a neurobiological correlate for distinguishing between similar environments. The inability to detect and then respond to contextual changes results in a process that has been dubbed the 'uncoupling of 
effect from external context',53 and the cognitive distortions produced by this uncoupling are thought to be associated with development of disturbed mood. Indeed, recent findings ${ }^{54,55}$ show a causal relationship between reduced neurogenesis and an anxiogenic phenotype and thus provide further evidence for links between adult neurogenesis and the ability to both integrate novel information into existing memory and temporally separate novel events. This theory would predict that in our paradigm NG- mice exposed to EE would be unable to uncouple the SC-acquired negative experiences, leading to perpetuation of the submissive phenotype. In contrast, stimulation of neurogenesis in Ctrl mice exposed to EE would facilitate coupling of positive affect with the new environmental context. As a result, Ctrl/EE mice would be rendered better able to detect and then capitalize on improvements in the environment, thus facilitating amelioration of submissive and depressive behaviors. In our model, Ctrl/EE mice showed a robust recovery from the SC-induced submissive phenotype when compared with both Ctrl/IE and NG-/EE mice, suggesting a role for neurogenesis in the extinction of the submissive, depressive-like state.

The current results support the hypothesis that impaired neurogenesis results in inefficient integration of novel stimulation, rendering the animal unable to distinguish changes in contextual and temporal information. Thus, newborn cells in the DG may be important for the animal to perceive a similar negative environment (SC), and to then adopt new coping strategies to combat it. The ability of EE to accelerate and enhance neurogenesis may be critical for this process. Furthermore, newborn DG cells may also be necessary for the animal to take advantage of the new contextual experiences provided by EE. If neurogenesis is impaired, existing DG cells must represent features of both the old and new environments as well as their emotional contexts, compromising the animal's ability to adapt to the new context with appropriate affect.

In conclusion, our study makes several important contributions. First, exposure to chronic psychosocial stress results in an ethologically relevant behavioral phenotype that resembles hallmarks of clinical depression in humans. Second, EE rescues the submissive phenotype and depressive-like behaviors adopted in response to chronic psychosocial stress, suggesting that EE increases stress resiliency and promotes adaptive coping. Third, these beneficial effects of EE are dependent on intact adult neurogenesis. These findings suggest that maladaptive responses to adverse psychosocial situations can be ameliorated by behavioral therapies, and that these improvements may be aided by promoting neurogenesis.

\section{Conflict of interest}

The authors declare no conflict of interest.

\section{Acknowledgments}

The work was supported by the Intramural Research Program of the National Institute of Mental Health, NIH. We thank JB Lednak, DV Jimenez and KM Cardinale for technical assistance and animal maintenance.

Author contributions: ML designed and conducted behavioral experiments. RJS-generated transgenic animals performed histology and conducted behavioral experiments. KM conducted histology and behavioral experiments. $\mathrm{MH}$ and HKM contributed to experimental design and interpretation. ML, RJS, $\mathrm{KM}$ and $\mathrm{MH}$ contributed to preparation of the article.

\section{References}

1 Nithianantharajah J, Hannan AJ. Enriched environments, experience-dependent plasticity and disorders of the nervous system. Nat Rev Neurosci 2006; 7: 697-709.

2 Fox C, Merali Z, Harrison C. Therapeutic and protective effect of environmental enrichment against psychogenic and neurogenic stress. Behavioural Brain Res 2006; 175: 1-8.

3 Caspi A, Sugden K, Moffitt TE, Taylor A, Craig IW, Harrington H et al. Influence of life stress on depression: moderation by a polymorphism in the 5-HTT gene. Science 2003; 301: 386-389.

4 Risch N, Herrell R, Lehner T, Liang KY, Eaves L, Hoh J et al. Interaction between the serotonin transporter gene (5-HTTLPR), stressful life events, and risk of depression: a meta-analysis. JAMA 2009; 301: 2462-2471.

5 Kozorovitskiy Y, Gould E. Dominance hierarchy influences adult neurogenesis in the dentate gyrus. J Neurosci 2004; 24: 6755-6759.

6 Gould E, McEwen BS, Tanapat P, Galea LA, Fuchs E. Neurogenesis in the dentate gyrus of the adult tree shrew is regulated by psychosocial stress and NMDA receptor activation. J Neurosci 1997; 17: 2492-2498.

7 Malberg JE, Eisch AJ, Nestler EJ, Duman RS. Chronic antidepressant treatment increases neurogenesis in adult rat hippocampus. J Neurosci 2000; 20: 9104-9110.

8 Sairanen M, Lucas G, Ernfors P, Castren M, Castren E. Brainderived neurotrophic factor and antidepressant drugs have different but coordinated effects on neuronal turnover, proliferation, and survival in the adult dentate gyrus. J Neurosci 2005; 25: 1089-1094.

9 Santarelli L, Saxe M, Gross C, Surget A, Battaglia F, Dulawa S et al. Requirement of hippocampal neurogenesis for the behavioral effects of antidepressants. Science 2003; 301: 805-809.

10 Surget A, Saxe M, Leman S, Ibarguen-Vargas Y, Chalon S, Griebel G et al. Drug-dependent requirement of hippocampal neurogenesis in a model of depression and of antidepressant reversal. Biol Psychiatry 2008; 64: 293-301.

11 Kempermann G, Kuhn HG, Gage FH. More hippocampal neurons in adult mice living in an enriched environment. Nature 1997; 386: 493-495.

12 Seri B, Garcia-Verdugo JM, McEwen BS, Alvarez-Buylla A. Astrocytes give rise to new neurons in the adult mammalian hippocampus. J Neurosci 2001; 21: 7153-7160.

13 Eriksson PS, Perfilieva E, Bjork-Eriksson T, Alborn AM, Nordborg C, Peterson DA et al. Neurogenesis in the adult human hippocampus. Nat Med 1998; 4: 1313-1317.

14 Greenwood BN, Fleshner M. Exercise, learned helplessness, and the stress-resistant brain. Neuromol Med 2008; 10: 81-98.

15 Laplagne DA, Esposito MS, Piatti VC, Morgenstern NA, Zhao C, van Praag $\mathrm{H}$ et al. Functional convergence of neurons generated in the developing and adult hippocampus. PLoS Biol 2006; 4: e409.

16 Waddell J, Shors TJ. Neurogenesis, learning and associative strength. Eur J Neurosci 2008; 27: 3020-3028.

17 Shors TJ, Miesegaes G, Beylin A, Zhao M, Rydel T, Gould E. Neurogenesis in the adult is involved in the formation of trace memories. Nature 2001; 410: 372-376.

18 Gould E, Beylin A, Tanapat P, Reeves A, Shors TJ. Learning enhances adult neurogenesis in the hippocampal formation. Nat Neurosci 1999; 2: 260-265. 
19 Winocur G, Wojtowicz JM, Sekeres M, Snyder JS, Wang S. Inhibition of neurogenesis interferes with hippocampus-dependent memory function. Hippocampus 2006; 16: 296-304.

20 Snyder JS, Hong NS, McDonald RJ, Wojtowicz JM. A role for adult neurogenesis in spatial long-term memory. Neuroscience 2005; 130: $843-852$.

21 Chapillon P, Manneche C, Belzung C, Caston J. Rearing environmental enrichment in two inbred strains of mice: 1. Effects on emotional reactivity. Behav Genet 1999; 29: 41-46.

22 Larsson F, Winblad B, Mohammed AH. Psychological stress and environmental adaptation in enriched vs impoverished housed rats. Pharmacol Biochem Behavior 2002; 73: 193-207.

23 Mohammed AH, Henriksson BG, Soderstrom S, Ebendal T, Olsson T, Seckl JR. Environmental influences on the central nervous system and their implications for the aging rat. Behavioural Brain Res 1993; 57: 183-191.

24 Pollak DD, Monje FJ, Zuckerman L, Denny CA, Drew MR, Kandel ER. An animal model of a behavioral intervention for depression. Neuron 2008; 60: 149-161.

25 Delaney CL, Brenner M, Messing A. Conditional ablation of cerebellar astrocytes in postnatal transgenic mice. J Neurosci 1996; 16: 6908-6918.

26 Garcia AD, Doan NB, Imura T, Bush TG, Sofroniew MV. GFAPexpressing progenitors are the principal source of constitutive neurogenesis in adult mouse forebrain. Nat Neurosci 2004; 7: 1233-1241.

27 Savidge TC, Newman P, Pothoulakis C, Ruhl A, Neunlist M, Bourreille A et al. Enteric glia regulate intestinal barrier function and inflammation via release of S-nitrosoglutathione. Gastroenterology 2007; 132: 1344-1358.

28 Mitchell PJ, Redfern PH. Animal models of depressive illness: the importance of chronic drug treatment. Curr Pharm Des 2005; 11: 171-203.

29 Southwick SM, Vythilingam M, Charney DS. The psychobiology of depression and resilience to stress: implications for prevention and treatment. Annu Rev Clin Psychol 2005; 1: 255-291.

30 Bennett EL, Diamond MC, Krech D, Rosenzweig MR. Chemical and Anatomical Plasticity Brain. Science 1964; 146: 610-619.

31 Volkmar FR, Greenough WT. Rearing complexity affects branching of dendrites in the visual cortex of the rat. Science 1972; 176: 1445-1447.

32 Swain RA, Harris AB, Wiener EC, Dutka MV, Morris HD, Theien $\mathrm{BE}$ et al. Prolonged exercise induces angiogenesis and increases cerebral blood volume in primary motor cortex of the rat. Neuroscience 2003; 117: 1037-1046.

33 Olsson T, Mohammed AH, Donaldson LF, Henriksson BG, Seckl JR. Glucocorticoid receptor and NGFI-A gene expression are induced in the hippocampus after environmental enrichment in adult rats. Brain Res 1994; 23: 349-353.

34 Droste SK, Chandramohan Y, Hill LE, Linthorst AC, Reul JM. Voluntary exercise impacts on the rat hypothalamic-pituitaryadrenocortical axis mainly at the adrenal level. Neuroendocrinology 2007; 86: 26-37.

35 Sasse SK, Greenwood BN, Masini CV, Nyhuis TJ, Fleshner M, Day $\mathrm{HE}$ et al. Chronic voluntary wheel running facilitates corticosterone response habituation to repeated audiogenic stress exposure in male rats. Stress (Amsterdam, Netherlands) 2008; 11: 425-437.

36 Brady LS, Lynn AB, Whitfield Jr HJ, Kim H, Herkenham M. Intrahippocampal colchicine alters hypothalamic corticotropinreleasing hormone and hippocampal steroid receptor mRNA in rat brain. Neuroendocrinology 1992; 55: 121-133.

37 Schloesser RJ, Manji HK, Martinowich K. Suppression of adult neurogenesis leads to an increased hypothalamo-pituitary-adrenal axis response. Neuroreport 2009; 20: 553-557.
38 Ribeiro SC, Tandon R, Grunhaus L, Greden JF. The DST as a predictor of outcome in depression: a meta-analysis. Am J Psychiatry 1993; 150: 1618-1629.

39 Linkowski P, Mendlewicz J, Kerkhofs M, Leclercq R, Golstein J, Brasseur M et al. 24-hour profiles of adrenocorticotropin, cortisol, and growth hormone in major depressive illness: effect of antidepressant treatment. J Clin Endocrinol Metab 1987; 65: 141-152.

40 Brown J, Cooper-Kuhn CM, Kempermann G, Van Praag H, Winkler J, Gage FH et al. Enriched environment and physical activity stimulate hippocampal but not olfactory bulb neurogenesis. Eur J Neurosci 2003; 17: 2042-2046.

41 Dranovsky A, Hen R. Hippocampal neurogenesis: regulation by stress and antidepressants. Biol Psychiatry 2006; 59: 1136-1143.

42 Madsen TM, Treschow A, Bengzon J, Bolwig TG, Lindvall O, Tingstrom A. Increased neurogenesis in a model of electroconvulsive therapy. Biol Psychiatry 2000; 47: 1043-1049.

43 van Praag H, Kempermann G, Gage FH. Running increases cell proliferation and neurogenesis in the adult mouse dentate gyrus. Nat Neurosci 1999; 2: 266-270.

44 David DJ, Samuels BA, Rainer Q, Wang JW, Marsteller D, Mendez I et al. Neurogenesis-dependent and -independent effects of fluoxetine in an animal model of anxiety/depression. Neuron 2009; 62: 479-493.

45 Vollmayr B, Simonis C, Weber S, Gass P, Henn F. Reduced cell proliferation in the dentate gyrus is not correlated with the development of learned helplessness. Biol Psychiatry 2003; 54: 1035-1040.

46 Meshi D, Drew MR, Saxe M, Ansorge MS, David D, Santarelli L et al. Hippocampal neurogenesis is not required for behavioral effects of environmental enrichment. Nat Neurosci 2006; 9: 729-731.

47 Meltzer LA, Yabaluri R, Deisseroth K. A role for circuit homeostasis in adult neurogenesis. Trends Neurosci 2005; 28: 653-660.

48 Wiskott L, Rasch MJ, Kempermann G. A functional hypothesis for adult hippocampal neurogenesis: avoidance of catastrophic interference in the dentate gyrus. Hippocampus 2006; 16: 329-343.

49 McHugh TJ, Jones MW, Quinn JJ, Balthasar N, Coppari R, Elmquis JK et al. Dentate gyrus NMDA receptors mediate rapid pattern separation in the hippocampal network. Science 2007; 317: 94-99.

50 Clelland CD, Choi M, Romberg C, Clemenson Jr GD, Fragniere A, Tyers $\mathrm{P}$ et al. A functional role for adult hippocampal neurogenesis in spatial pattern separation. Science 2009; 325: 210-213.

51 Aimone JB, Wiles J, Gage FH. Computational influence of adult neurogenesis on memory encoding. Neuron 2009; 61: 187-202.

52 Tashiro A, Makino H, Gage FH. Experience-specific functional modification of the dentate gyrus through adult neurogenesis: a critical period during an immature stage. J Neurosci 2007; 27: 3252-3259.

53 Perera TD, Park S, Nemirovskaya Y. Cognitive role of neurogenesis in depression and antidepressant treatment. Neuroscientist 2008; 14: 326-338.

54 Revest JM, Dupret D, Koehl M, Funk-Reiter C, Grosjean N, Piazza PV et al. Adult hippocampal neurogenesis is involved in anxiety-related behaviors. Mol Psychiatry 2009; 14: 959-967.

55 Bergami M, Rimondini R, Santi S, Blum R, Gotz M, Canossa M. Deletion of TrkB in adult progenitors alters newborn neuron integration into hippocampal circuits and increases anxiety-like behavior. Proc Natl Acad Sci USA 2008; 105: 15570-15575.

This work is licensed under the Creative Commons Attribution-NonCommercialNo Derivative Works 3.0 Unported License. To view a copy of this license, visit http://creativecommons. org/licenses/by-nc-nd/3.0/

Supplementary Information accompanies the paper on the Molecular Psychiatry website (http://www.nature.com/mp) 